\title{
TESTING OF ENERGY METERS UNDER THREE-PHASE DETERMINED AND RANDOM NONSINUSOIDAL CONDITIONS
}

\section{Andrzej Olencki, Piotr Mróz}

University of Zielona Góra, Faculty of Electrical Engineering, Computer Science and Electronics, Institute of Computer Engineering and Electronics, prof. Z. Szafrana 2, 65-516 Zielona Góra, Poland ( $\$ a.olencki@iie.uz.zgora.pl, +48 683282329$)$

\begin{abstract}
Electric energy meters are designed to account energy under sinusoidal and nonsinusoidal conditions, because both, old and new standards for energy meters require testing their accuracy under different conditions. The latest EN 50470 standard increases the range of meter testing under nonsinusoidal conditions, introducing new shapes of test signals such as the phase fired waveform or the burst fired waveform. This paper discusses calibration problems of electronic revenue energy meters for direct connection and for connection through current transformers, and it proposes a new calibration procedure which reproduces normal operating conditions better: three-phase configurations of measurement systems, load range during meter testing or shapes of test signals. Recently, modern Electrical Power Standards, also known as Power Calibrators, enable automatic testing of various types of electrical devices, including electricity meters in their normal operating conditions. This article presents examples of single and multi position fully automatic test systems, which employ Power/Energy Calibrator from Poland as the precision source with programmed waveforms of three phase voltages up to $560 \mathrm{~V}$ and currents up to 120 A conforming to EN 50470, or with random waveforms generated by PC software random wave generator. Measurement uncertainty of the energy meters under different nonsinusoidal conditions using a test system with reference to accuracy of the power calibrator or to the reference meter, are discussed.
\end{abstract}

Comparative analysis of test results for different shapes of voltage and current signals is presented in the conclusions of this paper.

Keywords: energy meter testing, nonsinusoidal conditions, energy calibrators, electrical power standards, automatic calibration procedure.

(C) 2014 Polish Academy of Sciences. All rights reserved

\section{Introduction}

Measuring electronic instruments should be tested under conditions as similar to the actual conditions of their operation as possible - this statement is obvious and does not require any justification. This applies in particular to electronic revenue electrical energy meters (EEM), whose reading is the reference for financial settlements between the supplier and the consumer of energy [1] - the latest directives state that the measuring instruments used in commercial transactions must be verified in the actual operating conditions [2].

An important group of tests on accuracy of EEMs includes verification requirements such as:

- limits of percentage error due to load variation under reference conditions,

- limits of additional percentage error due to the influence of quantities such as temperature variation, voltage variation and frequency variation,

- limits of disturbances effect such as, for example, harmonic components in the current and voltage circuits, odd harmonics in the current circuit, sub-harmonics in the current circuit. 
Especially the last group of tests reproduces actual operating conditions for EEMs. EEM testing under nonsinusoidal conditions has been in use for a long time [3, 4]. According to $[5,6]$ induction energy meters were tested on the influence of the $3^{\text {rd }}$ harmonic in the current at different values of phase angles between harmonics. From the experimental study [7] it has been concluded that induction meters with linear load or with current distortions below $20 \%$ are not obsolete for active energy measurement. More realistic test conditions were considered [8], by voltage and current harmonic content selection in accordance to the limits reported respectively in the EN 50160 [9] and the EN 61000-3-2 [10].

The latest standard EN 50470 [11] for active energy meters introduces several new tests of the EEM under nonsinusoidal conditions, such as testing the presence of the $5^{\text {th }}$ voltage and current harmonic, odd harmonics or sub-harmonics in currents. This standard requires the tests to be carried out only for balanced load at unity power factor, and only for one current value. It's difficult to say whether these parameters of the test points are representative for other conditions. It is also unclear what requirements should be met by the EEM for the other test points - single phase load with balanced voltages (unbalanced load), other than unit power factor and other current values.

There is an ongoing search for the best shapes of test signals in the scientific literature, for the EEM calibration under nonsinusoidal conditions. In [12] it is proposed to generate arbitrary signals with known harmonic content of shapes such as triangular, square or trapezoidal ones - the results of the performed simulations do not seem to indicate any preferred waveform of this shape.

Next article [13] proposes application of randomly distorted waveforms for voltage and current signals with total harmonic distortion (THD) factor equal to $10 \%$ for voltage and $40 \%$ for current. These values are equal to the ones applied in the $5^{\text {th }}$ harmonic presence test according to the EN 50470. Unfortunately, the EEM testing was performed under unrealistic conditions - the three-phase EEM was tested with a single phase source. The question remains whether the results of the three-phase meter research with single-phase configuration allows one to draw conclusions about the suitability of those test signals.

There is no doubt that the EN 50470 has determined a lot of clear advice on how to test the EEM, but there is some uncertainty whether such conditions are sufficiently representative. Some important aspects of this problem are dealt with in this article. In particular, the paper focuses on a test method of the EEM based on the generation of calibrated test signals with known waveform or pre-assigned harmonic content. The critical point, with this method, is the comparison of the test results for each kind of the test signals. Therefore, this paper focuses on finding out which test is the most difficult one to meet the EEM requirements.

\section{PROPOSED PROCEDURE}

The proposed solution to the EEM testing under real working conditions is based on generation of determined waveforms of voltage and/or current signals according to the EN 50470 and the randomly distorted signals covering full current range and in different configurations. Such an experiment should allow for validation of some approaches previously proposed in the field literature and standards.

General knowledge of metrology and the analysis of standard requirements, reveal the need for at least triple measurement repetition of each required test point, and the need to calculate the standard deviation. The repeatability at any test point shall be better than $1 / 10^{\text {th }}$ of the percentage error limit in each test point [11]. In order to achieve that, the operator shall set the necessary number of pulses or measurement time value.

It is important to ensure proper configuration of the measurement - especially for threephase meters, which operate in the real conditions with balanced load or with a single-phase 
load, but with balanced three phase voltages applied to the voltage circuits (unbalanced load). Testing the three-phase meter with single-phase voltage and current sources, can result in significant errors. Therefore, testing the three-phase meters should be performed with threephase voltage and current sources, and the possibility to operate in the configurations with balanced load and with unbalanced load.

Meter testing should be carried out within the full range of load at the specified percentage error and within which the meter actually works. The EN 50470 standard specifies the percentage error in the range from $I_{\min }$ (minimum current) up to $I_{\max }$ (maximum current). The EEM for connection through current transformers has a recommended range of values $I_{t r}$ transitional current equal to 0.05-0.1-0.25 A. Having taken into account that the minimum current is expressed as $I_{\min } \leq 0.3 \cdot I_{t r}$, this gives the minimum current value $0,3 \cdot 0,05 \mathrm{~A}=0,015 \mathrm{~A}$. The EEM for direct connection has a recommended range of values $\mathrm{I}_{\text {tr }}$ transitional current equal to 0.5-1-1.5-2 A. Taking into account the fact that the maximum current is expressed as $I_{\max } \geq 50 \cdot I_{t r}$, which provides the current values $25-50-75-100 \mathrm{~A}$, the maximum three-phase current source should be at least 50.2 A=100 A.

Real energy network parameters are variables of time and therefore the standard specifies limits of additional percentage errors due to the influence of voltage variation $\pm 10 \%$ of $U_{n}$ and frequency variation $\pm 2 \%$ of $\mathrm{f}_{\mathrm{n}}$, where $U_{n}$ is the reference (rated) voltage and $\mathrm{f}_{\mathrm{n}}$ is the reference (rated) frequency. Therefore RMS values of the voltage signal can be set to any value in the range from $0.9 \cdot U_{n}$ to $1.1 \cdot U_{n}$ and the fundamental frequency $f_{l}$ can be set to any value in the range from $0.98 \cdot f_{n}$ to $1.02 \cdot f_{n}$. The power factor (or the phase shift) with respect to the fundamental component of the voltage and current signal, can be set to any value in the range $0.5 \mathrm{~L}$ (ind) ...1...0.8C (cap).

Having taken the following constraints into account, there are five energy meter accuracy tests to be performed under the following conditions:

1. At reference conditions according to the EN 50470-3 with sinusoidal voltages and currents waveform - the test marked as "SIN test",

2. In the presence of harmonics according to the EN 50470-3 with the content of the $5^{\text {th }}$ harmonic voltage $U_{5}=10 \%$ of $U_{n}$ and of the $5^{\text {th }}$ harmonic current $I_{5}=40 \%$ of fundamental current. In this test marked as " $5 \mathrm{H}$ test", the harmonic power factor equals $\cos \varphi_{5}=1$, the fundamental and harmonic voltages are in phase at positive zero crossing,

3. In the presence of odd harmonics in the currents. This test marked as "PFW test" shall be performed with the Phase Fired waveform fired at 1/4 and 3/4 of the period in the current circuits according to the EN 50470-3,

4. In the presence of sub-harmonics in the currents. This test marked as "BFW test" shall be performed with the Burst Fired waveform 2 cycles on 2 cycles off in the current circuits according to the EN 50470-3,

5. Randomly distorted waveforms of voltages and currents. In this test marked as "RH test", a random number of harmonic components, which are randomly distributed between the fundamental component and the maximum required harmonic (set to the $20^{\text {th }}$ one according to [9]), are generated. Amplitude and phase shift are randomly generated for each of the harmonic components, determined in the previous step. The current waveform has the same harmonic components as the voltage waveform. A check is performed, so that the THD factor in voltages is equal to $10 \%$ and THD factor in currents is equal to $40 \%$.

It is suggested for all tests to be performed within the full range of load currents for current values $I_{\min }, I_{t r}, 5 \cdot I_{t r}, 10 \cdot I_{t r}, 0.5 \cdot I_{\max }$. Providing the same conditions for these different tests is necessary to compare the sharpness of individual tests, and to answer the question whether the conditions of the tests proposed in EN 50470 are optimal, the requirement that the tests PFW and $\mathrm{BFW}$ are to be performed only for the current $5 \cdot \mathrm{I}_{t r}$ is questionable, the $5 \mathrm{H}$ test is to be 
implemented for the current $0.5 \cdot I_{\max }$ (see Table I). In the case of testing the meter for direct connection with nominal current value equal to 10 A with $1000 \%$ overload, the difference between these currents is up to ten times, because $5 \cdot I_{t r}=5 \mathrm{~A}$ and $0.5 \cdot I_{\max }=50 \mathrm{~A}$.

Table 1. Test Points for Accuracy Testing of Energy Meters Acc. to the EN 50470.

\begin{tabular}{|c|c|c|c|c|c|c|c|c|c|}
\hline \multirow{3}{*}{ Load } & \multirow[t]{3}{*}{ U } & \multirow[t]{3}{*}{$f$} & \multirow{3}{*}{$\begin{array}{c}\text { Power } \\
\text { Factor } \\
\text { (PF) }\end{array}$} & \multicolumn{6}{|c|}{$\mathrm{I}=$} \\
\hline & & & & $I_{\min }$ & $I_{\text {tr }}$ & $5 \cdot \cdot_{\text {tr }}$ & 10. $I_{\text {tr }}$ & $0.5 \cdot I_{\max }$ & $I_{\max }$ \\
\hline & & & & $0,5 \mathrm{~A}$ & $1 \mathrm{~A}$ & $5 \mathrm{~A}$ & $10 \mathrm{~A}$ & $50 \mathrm{~A}$ & $100 \mathrm{~A}$ \\
\hline L123 & $U_{n}$ & $f_{n}$ & 1 & SIN & SIN & $\begin{array}{l}\text { PFW } \\
\text { BFW }\end{array}$ & SIN & $5 \mathrm{H}$ & SIN \\
\hline L123 & & & $0,5 \mathrm{~L}$ & & SIN & & SIN & & SIN \\
\hline L123 & & & $0,8 \mathrm{C}$ & & SIN & & SIN & & SIN \\
\hline $\mathrm{L} 1$ & & & 1 & & $\overline{\mathrm{SIN}}$ & & SIN & & SIN \\
\hline L1 & & & $0,5 \mathrm{~L}$ & & SIN & & SIN & & SIN \\
\hline L2 & & & 1 & & $\overline{\mathrm{SIN}}$ & & SIN & & SIN \\
\hline $\mathrm{L} 2$ & & & $0,5 \mathrm{~L}$ & & SIN & & SIN & & SIN \\
\hline$\overline{L 3}$ & & & 1 & & $\overline{S I N}$ & & SIN & & SIN \\
\hline L3 & & & $0,5 \mathrm{~L}$ & & SIN & & SIN & & SIN \\
\hline
\end{tabular}

For making tests described in Table 1, precision sources with programmed waveform of three-phase voltages and currents are used. The so-called Three Phase Power Calibrator or Electrical Power Standard has six outputs and can generate AC voltage and current at the same time. Also, the phase angle between them can be set with high accuracy, so we are given the possibility to simulate power. The six channels version can generate three voltages shifted usually by $120^{\circ}$ and $240^{\circ}$ or with the programmed values of angles between voltages and three currents shifted according to the voltages, plus an additional phase shift between the voltage and the current. Such systems like ROTEK 8100, FLUKE 6100A or CALMET C300 allow for a three-phase power network simulation. If the simulation power accuracy with such a system is inadequate, a three-phase reference meter can be used additionally, it is produced by several firms, for example RADIAN, ZERA or MTE. In his case, the accuracy requirements for energy are moved from the generating system to the reference measurement system - reference meter.

\section{TEST SYSTEM}

According to the considerations in the previous section, a three-phase test system has been implemented according to the schematics shown in Fig. 1 with reference to the power calibrator and in Fig. 2 with reference to the reference meter. The test system is controlled by a personal computer with Random wave generator PC soft and Calpro 300 PC soft (Fig. 1) or Calpro 300 MPX PC soft (Fig. 2). 


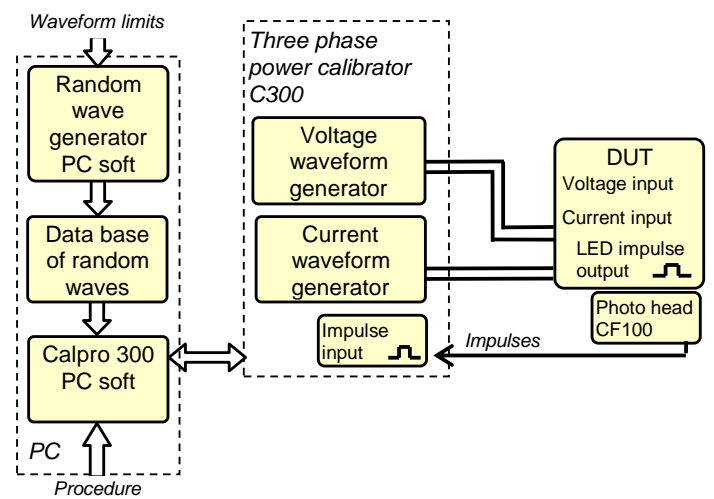

Fig. 1. Block diagram of the realized single position fully automatic test system for one Device Under Test (DUT) with reference to the power calibrator.

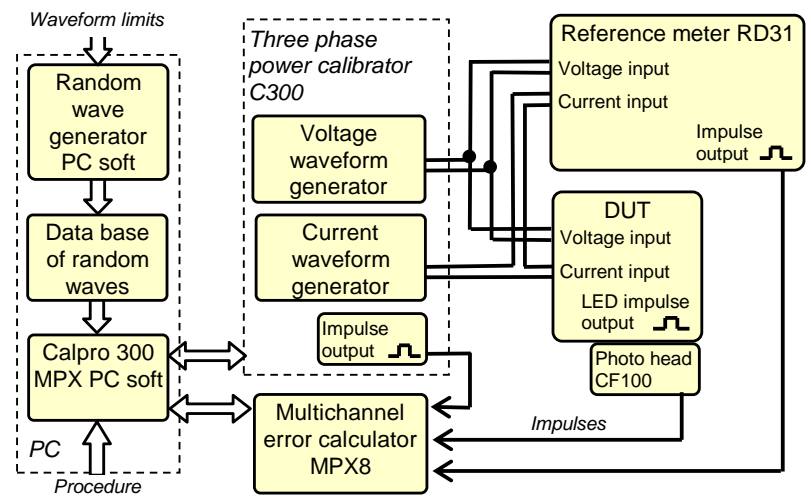

Fig. 2. Block diagram of the realized multi position fully automatic test system for simultaneous testing of several energy meters with reference to the power calibrator or the reference meter.

Three-phase voltages and currents with accurately programmed waveforms are generated by means of the power/energy calibrator, featuring fully independent control of voltages up to $560 \mathrm{~V}$ and currents up to $120 \mathrm{~A}$ during the tests. The voltage and current terminals of the calibrator are connected respectively with the voltage and current terminals of the DUT (Fig. 1) or the DUT and reference meter RADIAN RD31 (Fig. 2). A LED impulse output of the DUT is connected via a photo scanning head CALMET CF100 to the C300 impulse input (Fig. 1) or to a multi-channel error calculator CALMET MPX8 (Fig. 2). The remaining inputs of the multiplexer are connected to the impulse output of the reference meter and additionally to the impulse output of the power calibrator for monitoring the calibrator's accuracy in relation to the reference meter.

The active energy measured by the DUT is evaluated by counting a specified number of pulses provided by the DUT within a time interval controlled by the power calibrator. The time interval (or the number of pulses to be counted) is selected for each test in order to achieve a standard deviation on the measurement of the percentage error below $0.02 \%$ in the system with reference to the power calibrator (Fig.1) and below 0.002\% in the system with reference to the reference meter (Fig.2) in each test point - see chapter IV with experimental performance evaluation. Fig. 3 shows the implementation of the proposed single position fully automatic test systems, which are connected to the active energy meter mounted on a test rack.

Fig. 4 shows the front panel of the Three-phase random waveform generator PC soft. This program realized the virtual random wave generator implemented for generating random test 
signals and recording them to the Data base of random waves. The main window is divided into four sections: input data, harmonic data, calculated data and diagrams.

Input data are used to determine the parameters of the generated wave forms such as the value of voltage and current, THD distortion factor and number of the highest harmonic component. The maximal number of harmonic is set to 60 . Additionally, it is possible to set the voltage and current signals which should be distorted, the signals which must remain the same and the signals for which there should be randomly generated harmonic components.

The harmonic data shows parameters values for generated waveforms. This data consists of amplitude and phase values for each voltage and current harmonic component. The calculated data shows the voltage and current values and THD distortion factor calculated from the generated waveforms. In the main window there are two diagrams. The upper one shows the generated voltage and current waveform for one phase. The lower one shows the amplitude and phase of each harmonic component. Three buttons below the diagrams are used to change the displayed phase.

Virtual random wave generator randomly generates: a number of harmonic components, a number of harmonics and their amplitudes and phases. The generated waveforms are linearly corrected according to the defined THD distortion factors.

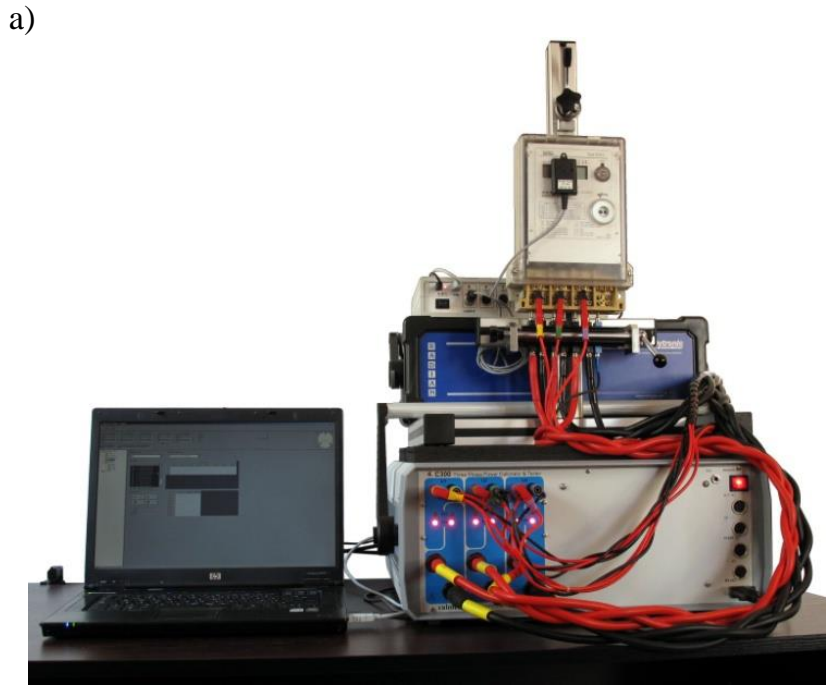

b)

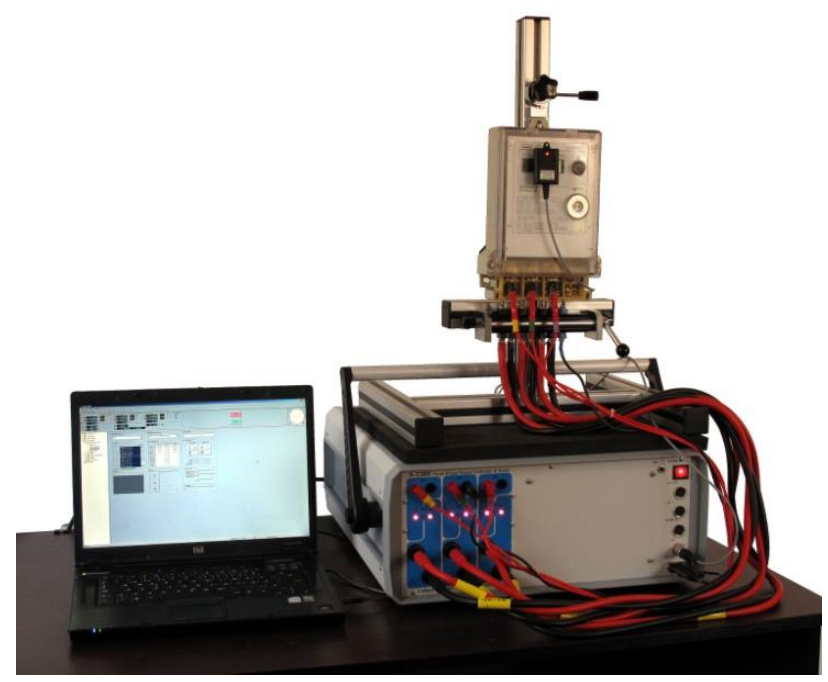

Fig.3. Photos of experimental bench:

a) for calibrations with 0.05 accuracy class and with reference to the reference meter acc. to the scheme Fig. 1 b) for calibrations with 0.005 accuracy class and with reference to the reference calibrator acc. to the scheme Fig. 2 
The generated waveforms can be saved to the "Data base of random waves". This data base is an additional library of shapes for Calpro 300 PC soft (Fig. 1) and Calpro 300 MPX PC soft (Fig. 2) software. The C300 calibrator, controlled by this software, can physically generate waveforms from the Data base of random waves.

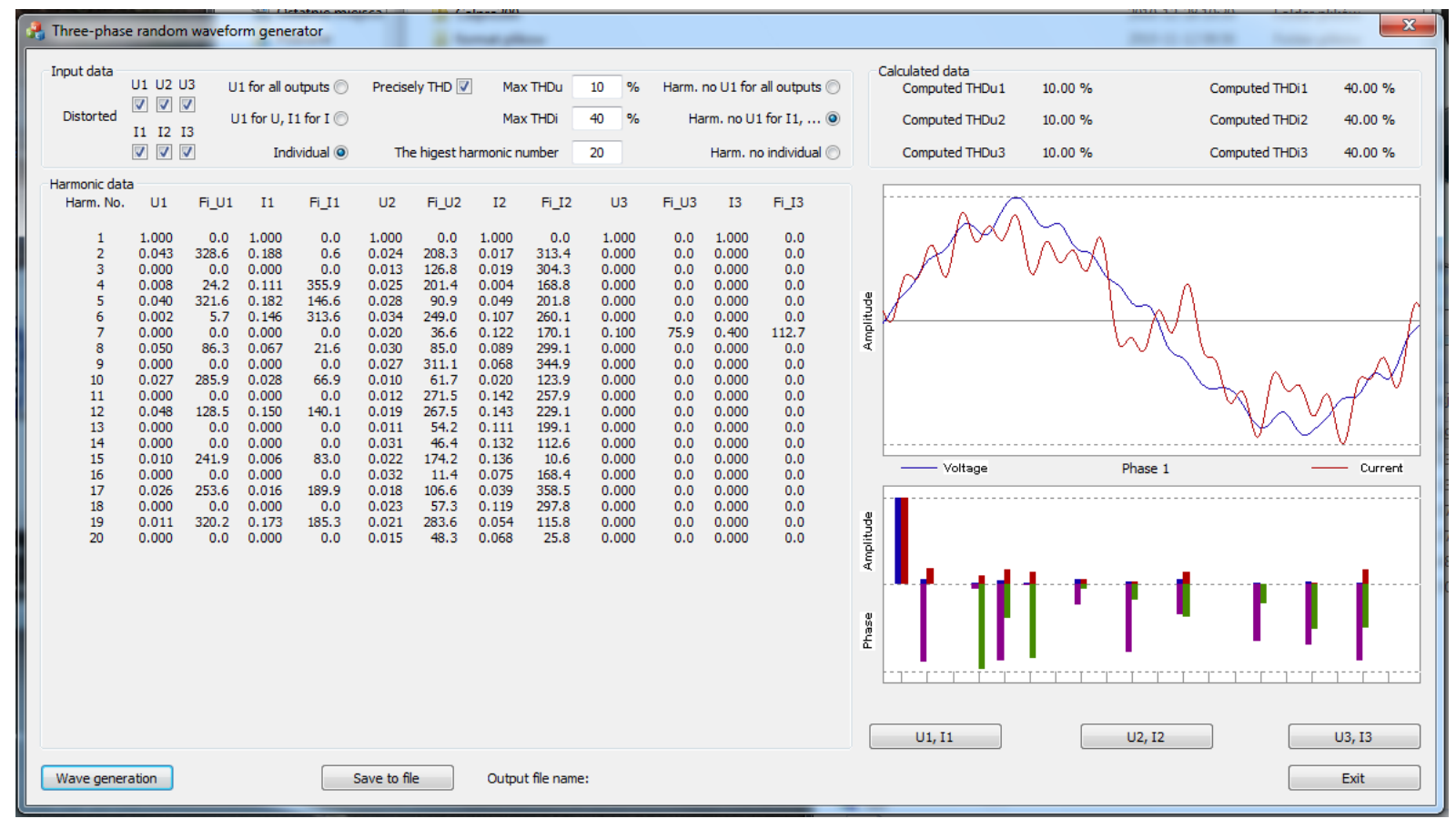

Fig. 4 The screenshot of the front panel of the realized virtual random wave generator.

Fig. 5 shows the implemented Procedure window of the Calpro 300 PC soft, it's purpose is to collect data for measuring procedures (the example shows the procedure for the RH test number 09). The waveform checkbox enables loading a file with randomly distorted waveforms of signals from the database of random waves created with the random wave generator PC soft, or from the database of special shapes created with the Calpro300 PC soft.

The measurement time in the field labeled "Test method / Time" equals 3s. In order to calculate the standard deviation, the number of measurements to perform in the field "Test duration / cycles" is equal to three replicates for each testing point.

\section{EXPERIMENTAL PERFORMANCE EVALUATION}

The expanded percentage uncertainty $U$ for a directly measured error of the DUT in the realized test system (Fig. 1), when the uncertainties of type A and type B are taken into consideration, results from

$$
U=k \cdot u_{C}=k \cdot \sqrt{u_{A}^{2}+u_{B}^{2}} .
$$

where $k$ is the coverage factor and $u_{C}$ is the combined standard uncertainty. 


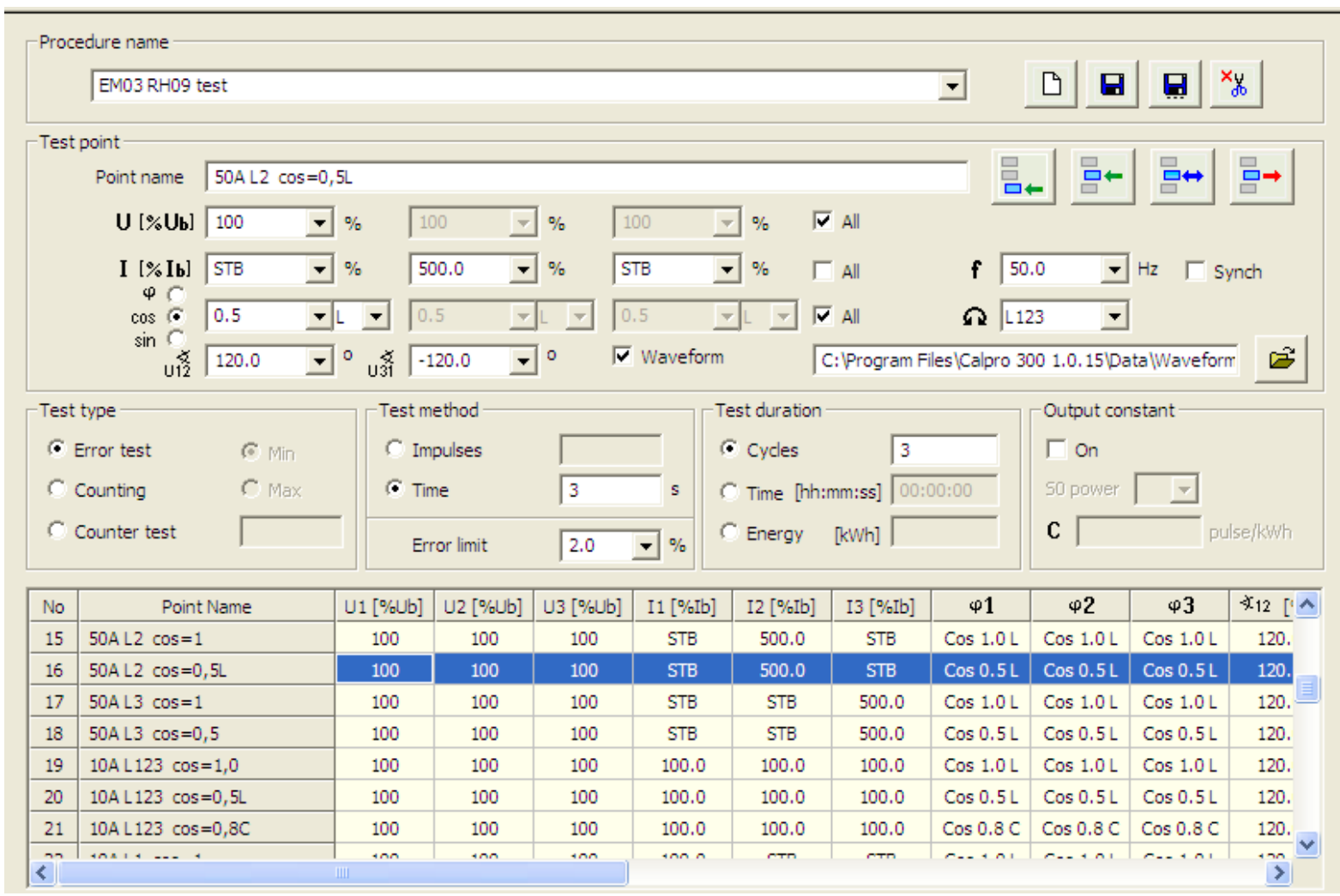

Fig. 5. The screenshot of the implemented Procedure window.

The standard uncertainty of type $\mathrm{A}-u_{A}$ is calculated from the observed scatter of the DUT's errors in the measurements series for each test point and may be expressed as the estimation of standard deviation

$$
u_{A}=s=\sqrt{\frac{1}{n-1} \sum_{i=1}^{n}\left(E_{i}-\bar{E}\right)^{2}} .
$$

where $n$ is a number of measurements for each test point, $E_{i}$ is the error percentage [11] in relation to the measurement value for the $i^{\text {th }}$ measurement point and $\bar{E}$ is the average value of the percentage error. The standard uncertainty of type A should be particularly taken into account in situation if it is need efficient energy meters verification, which requires energy measurement in a relatively short time. The shortening the test time increases the value of the standard deviation. The standard deviation value depends mainly on the DUT output impulses irregularities and fluctuations of source signals, so the standard deviation value is a random function of time and takes the values from the interval $\left[0 ; s_{\max }\right]$. What is a novelty in this analysis is the maximum value of standard deviation $s_{\max }$ being accounted for in the uncertainty calculation for electricity meters directly measured errors.

The [14] describes the test system and method which gives the opportunity to obtain the characteristics of the maximum value of standard deviation $s_{\max }$ as the function of energy measurement time $t_{M}$ in a fully automatic way. For the EM03 energy meter used as the DUT, this characteristic is presented in Fig. 6 . In order to acquire the value $s_{\max }<0.02 \%$, which, according to the IEC 736, is required for a Meter Testing Equipment (MTE) for testing the DUT accuracy class 1.0, the value of the measurement time satisfies the following condition

$$
t_{M} \geq 3 s \cap N \geq 1 \text {. }
$$


where $N$ is a number of counted pulses after the start pulse. Adaptation of this condition (3) allows one to obtain standard uncertainty of type $\mathrm{A}-u_{A}<0.02 \%$. Many authors ${ }^{13}$ do not apply standard uncertainty of type A at uncertainty evaluation of energy meters test results - this leads to unreasonable measurement time increase or to test results not meeting the required quality.

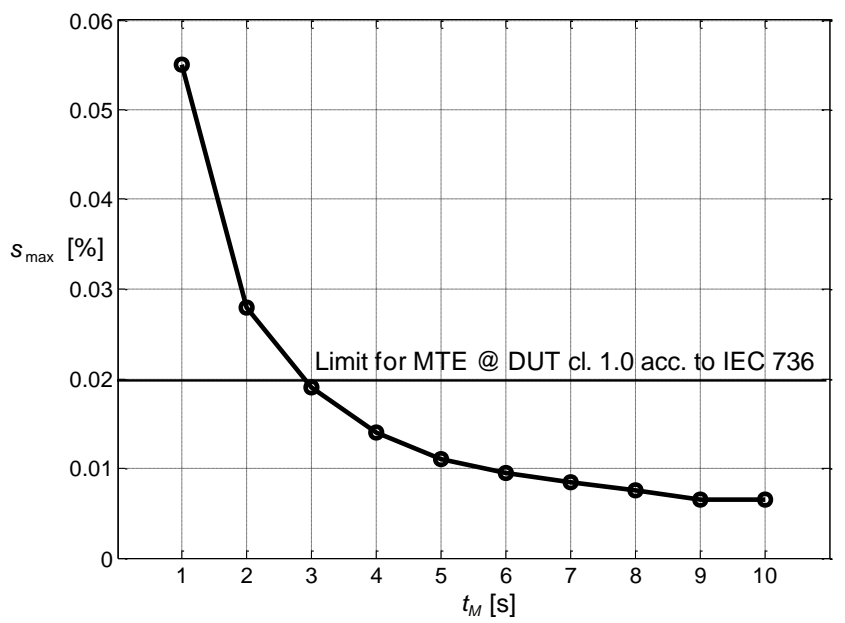

Fig. 6. The chart of the standard deviation estimation as energy measurement time for the EM03 energy meter.

Standard uncertainty of type $\mathrm{B}-u_{B}$ is equal to standard deviation of the assumed distribution of the C300 Calibrator errors. With the assumption that the C300 Calibrator errors have normal (Gaussian) distribution within the limits of maximum error $\Delta_{\mathrm{C}}$, standard uncertainty of type B under $\cos \varphi=1$ is equals

$$
u_{B}=\frac{\Delta_{C}}{3}=\frac{0.05 \%}{3}=0.017 \% \text {. }
$$

and for $\cos \varphi \neq 1$ with linear rise to $0.1 \%$ for $\cos \varphi=0.5$.

Therefore expanded uncertainty of designation the DUT error value for coverage factor $k=2$ and confidence level equal to $95 \%$ is calculated below

$$
U=2 \cdot \sqrt{(0.02 \%)^{2}+(0.017 \%)^{2}}=0.052 \%,
$$

for $\cos \varphi=1$ and $0.204 \%$ for $\cos \varphi=0.5$.

The performance of the whole test system, as far as strict metrology is concerned, is fully set according to:

- properly selected energy measurement time,

- performance of the power calibrator (scheme in Fig. 1) or the reference meter (scheme in Fig. 2).

In order to reduce uncertainty resulting from the DUT error value, the system with the reference meter (Fig. 2) should be used. Standard uncertainty of type B in this system equals $0.005 \% / 3=0.0017 \%$ and depends on the RD31 Reference Meter errors. It is clear that standard uncertainty of type A should also be reduced by extending the measurement time to about 
30-60 s, because $s_{\max } \approx \mathrm{f}\left(1 / t_{M}\right)$ according to Fig. 6 . The extended uncertainty for this system is about $0.0052 \%$ for $\cos \varphi=0.5 \ldots 1$.

\section{EXPERIMENTAL RESULTS}

Additional percentage error due to long duration disturbances (long duration disturbances effect) is defined as:

where:

$$
d E=d E_{N O N S I N}-d E_{S I N}
$$

$d E \quad$-additional percentage error due to long duration disturbances,

$d E_{\text {NONSIN }}-\quad$ meter error for nonsinusoidal waveform,

$d E_{S I N} \quad$-meter error for sinusoidal waveform

(reference conditions).

The EEM additional error research was carried out in nonsinusoidal conditions described in section II and measured with the uncertainty described in section IV. The three-phase fourwire active energy meter LUMEL EM03 [15] was used. This meter belongs to accuracy class B according to EN 50470 standards which corresponds to class 1 described in earlier standards. It is equipped with an impulse output with meter constant 1000 pulses $/ \mathrm{kWh}$. The signal from this output can be used to count energy by calibrator during accuracy testing. All tests were performed with preset energy measurement time equal to $3 \mathrm{~s}$.

The EEM was tested under specified sinusoidal and nonsinusoidal conditions in the circuit shown on Fig. 1. In this case the random wave generator PC soft block and the data base of random waves block are not used.

The EEM error for sinusoidal waveforms for balanced and unbalanced loads for unity power factor has been presented in Fig. 7. The difference between the percentage errors when the EEM is carrying a single-phase load and a balanced polyphase load at $I_{\text {ref }}(I=10 \mathrm{~A}$ for DUT) and unity power factor shall not exceed $1.5 \%$. For the tested EEM, the differences of errors are about $0.1 \%$. Therefore the chosen EEM is correctly adjusted and it serves the EEM additional errors testing very well.

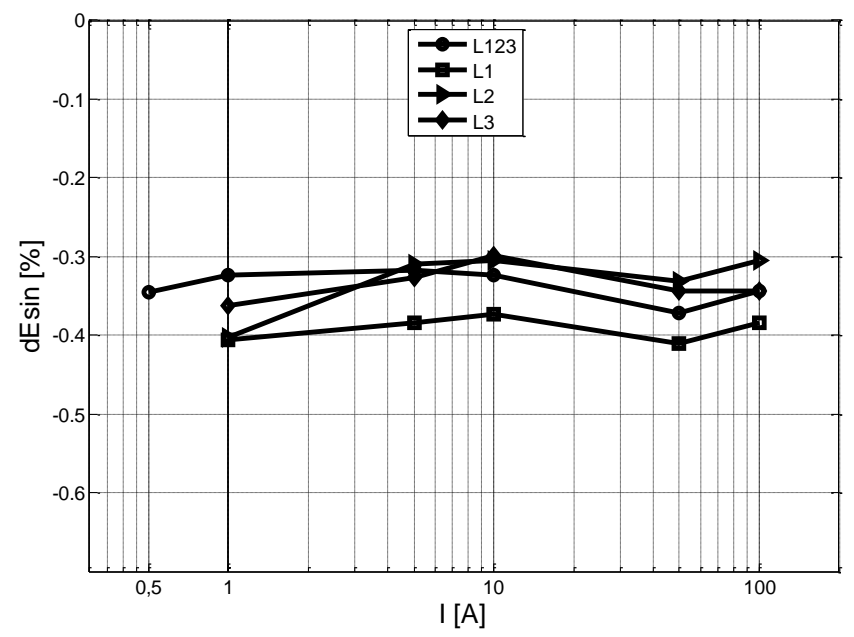

Fig. 7. The characteristics of the EEM error for sinusoidal waveforms (curve L123 for $\mathrm{PF}=1 \cap$ bal., curves L1, L2, L3 for $\mathrm{PF}=1$ กunbal.).

The summary of test results has been presented in Table II. It shows the additional error limitations (for 5H, PFW and BFW tests) and the DUT additional error values for the required 
conditions (unity power factor and balanced load and current value $50 \mathrm{~A}$ for $5 \mathrm{H}$ test and $5 \mathrm{~A}$ for PFW and BFW tests), and maximum additional error values $\max |d E|$ measured during tests in the range from $0.5 \mathrm{~A}$ to $50 \mathrm{~A}$ for unity power factor, or power factor equal to $0.5 \mathrm{~L}$ and balanced or unbalanced load.

The test results for the required conditions were within the limits. The additional error for PFW test was $0.1 \%$ at limit $3.0 \%$. In this case the error value is thirty times smaller than the limit. The EEM additional error for BFW test is $2.0 \%$ at limit $3.0 \%$. In this case the error margin is small. In the light of these results it can be stated that the BFW test is more demanding than the $5 \mathrm{H}, \mathrm{PFW}$ and $\mathrm{BFW}$ tests, and that the PFW test is the easiest test under the required conditions.

The maximum additional error values $\max |d E|$ in current ranging from $0.5 \mathrm{~A}$ to $50 \mathrm{~A}$ are higher than those in the results for the required conditions. The maximum additional error value is the one for BFW test for unity power factor and unbalanced load. This error value is three times higher than the limit. Almost all of maximum additional error values measured for power factor equal to $0.5 \mathrm{~L}$ are bigger than the values measured for unity power factor. In test $5 \mathrm{H}$ the maximum additional error values measured at power factor equal to $0.5 \mathrm{~L}$ exceed by $0.8 \%$ the limit given for the required conditions.

Fig. 8 shows the additional error for 5H, PFW and BFW tests for unity power factor and balanced load. The error curves are flat in full current range, so the EEM additional error can be tested at one current value.

Table 2. Maximum values of DUT's additional errors.

\begin{tabular}{|c|c|c|c|c|c|c|c|}
\hline \multirow{3}{*}{ Test } & \multicolumn{7}{|c|}{$\max \mid d E \quad[\%]$} \\
\hline & \multicolumn{3}{|c|}{$\begin{array}{l}\text { for the required } \\
\text { conditions } \\
\mathrm{PF}=1 \cap \text { bal. } \cap \mathrm{l}\end{array}$} & \multicolumn{4}{|c|}{ in current range $0.5-50 \mathrm{~A}$ for } \\
\hline & limit & result & $\mathrm{I}[\mathrm{A}]$ & $\begin{array}{l}\mathrm{PF}=1 \\
\cap \text { bal. }\end{array}$ & $\begin{array}{c}\text { PF=1 } \\
\text { nunbal. }\end{array}$ & $\begin{array}{c}\mathrm{PF}=0.5 \mathrm{~L} \\
\cap \text { bal. }\end{array}$ & $\begin{array}{l}\mathrm{PF}=0.5 \mathrm{~L} \\
\cap \text { unbal. }\end{array}$ \\
\hline $5 \mathrm{H}$ & 0.8 & 0.2 & 50 & 0.4 & 0.5 & 3.7 & 4.0 \\
\hline PFW & 3.0 & 0.1 & 5 & 0.8 & 0.7 & 1.0 & 1.0 \\
\hline BFW & 3.0 & 2.0 & 5 & 2.0 & 9.0 & 3.0 & 3.0 \\
\hline $\mathrm{RH}$ & $\mathrm{nd}^{*}$ & - & $\mathrm{nd}^{*}$ & 1.2 & 3.0 & 3.0 & 5.0 \\
\hline
\end{tabular}

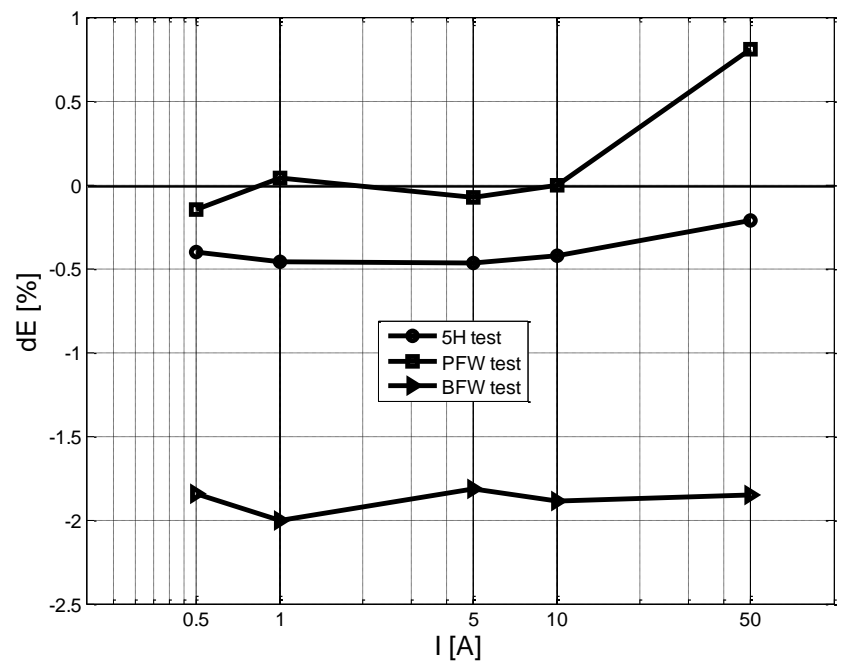

Fig. 8. The characteristics of the additional error for $5 \mathrm{H}, \mathrm{PFW}$ and $\mathrm{BFW}$ tests $(\mathrm{PF}=1 \cap$ bal. $)$. 
Fig. 9 shows the EEM additional errors for test 5H with balanced load (curve L123) and unbalanced load (curves L1, L2 and L3) at unity power factor. The maximum error differences between each curve are nearly $0.1 \%$.

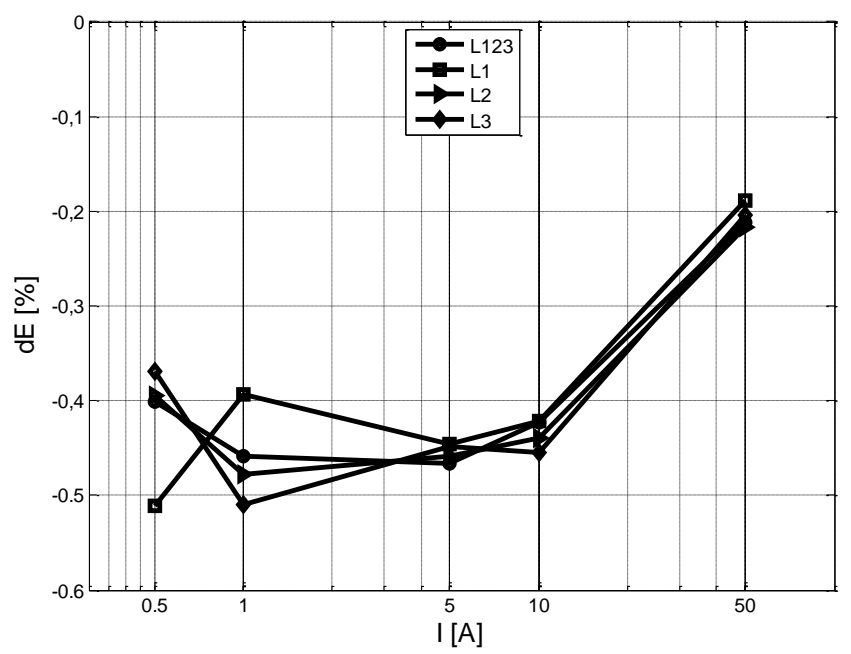

Fig. 9. The characteristics of the EEM additional error for $5 \mathrm{H}$ test $(\mathrm{PF}=1 \cap$ bal. $(\mathrm{L} 123) \cap$ unbal. $(\mathrm{L} 1, \mathrm{~L} 2, \mathrm{~L} 3))$.

Fig. 10 shows the EEM additional errors for BFW test with balanced load (curve L123) and unbalanced load (curves L1, L2 and L3) at unity power factor. The error values for L123, L2 and L3 confine themselves to the 3\% limit for BFW test with unity power factor, balanced load and current value $5 \mathrm{~A}$. The L1 phase additional errors exceed this limit. The maximum error difference between phase L1 and L3 amounts to nearly $11 \%$.

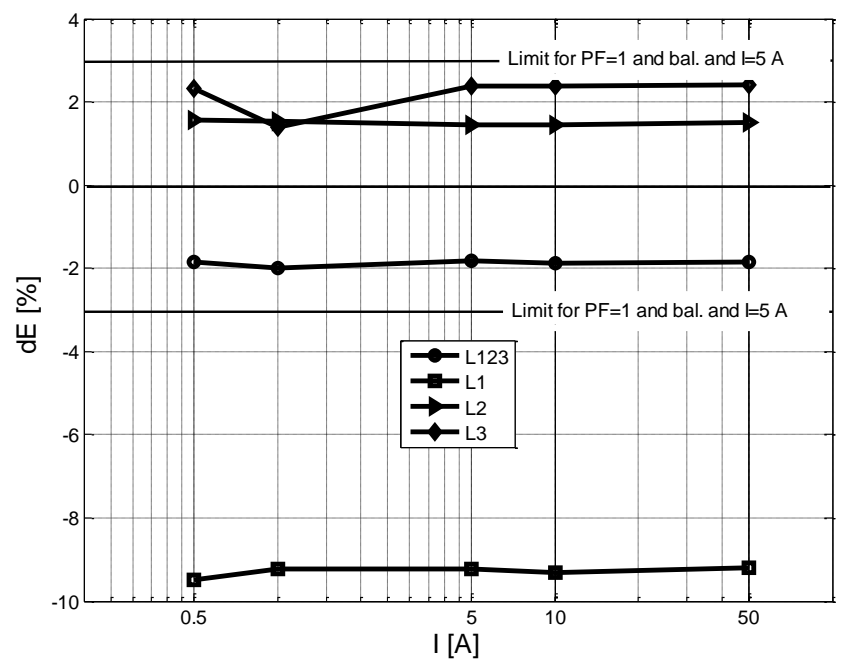

Fig. 10. The characteristics of the EEM additional error for BFW test $(\mathrm{PF}=1 \cap$ bal. $(\mathrm{L} 123) \cap$ unbal. $(\mathrm{L}, \mathrm{L} 2, \mathrm{~L} 3))$.

Fig. 11 shows the EEM additional error for $5 \mathrm{H}$ test with power factor equal to $0.5 \mathrm{~L}$ and balanced load (curve L123) and unbalanced load (curves L1, L2 and L3). There are also limits of additional error for $5 \mathrm{H}$ test marked with unity power factor and balanced load and current 50 A. The EEM additional error values in $5 \mathrm{H}$ test for unity power factor (Fig. 9) are confined 
to the $0.8 \%$ limit, but for power factor equal to $0.5 \mathrm{~L}$ the EEM additional error values are shifted by approximately 4\% (Fig. 11).

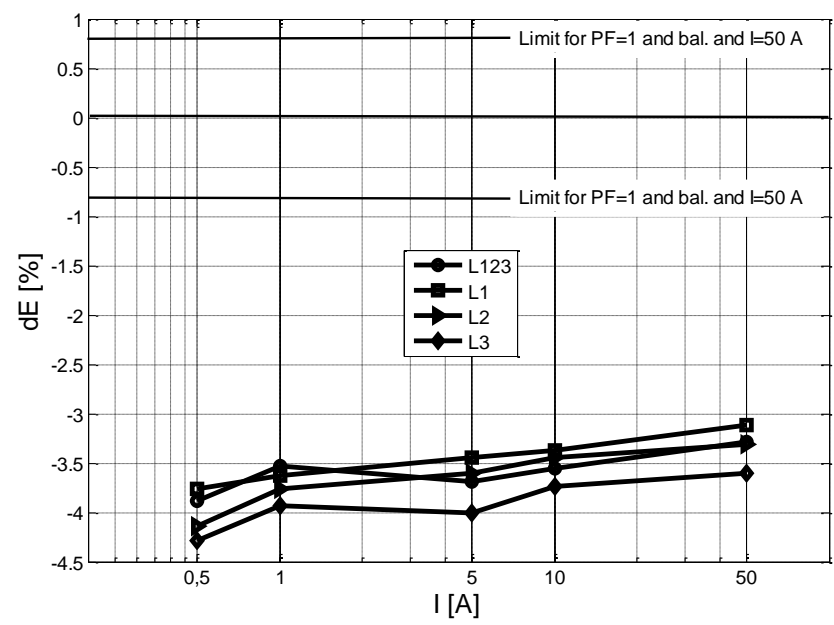

Fig. 11. The characteristics of the EEM additional error for $5 \mathrm{H}$ test $(\mathrm{PF}=0.5 \mathrm{~L} \cap$ bal.(L123) $\cap$ unbal.(L1, L2, L3).

RH tests were carried out for ten randomly generated signals. Those waveforms were generated by the Three-phase random waveform generator PC soft described in chapter III. Fig. 12 shows the EEM additional error for RH7 test with unity power factor and balanced load (curve L123) and unbalanced load (curves L1, L2 and L3). In this configuration the maximum error difference between phase L1 and L3 amounts to nearly $2 \%$.

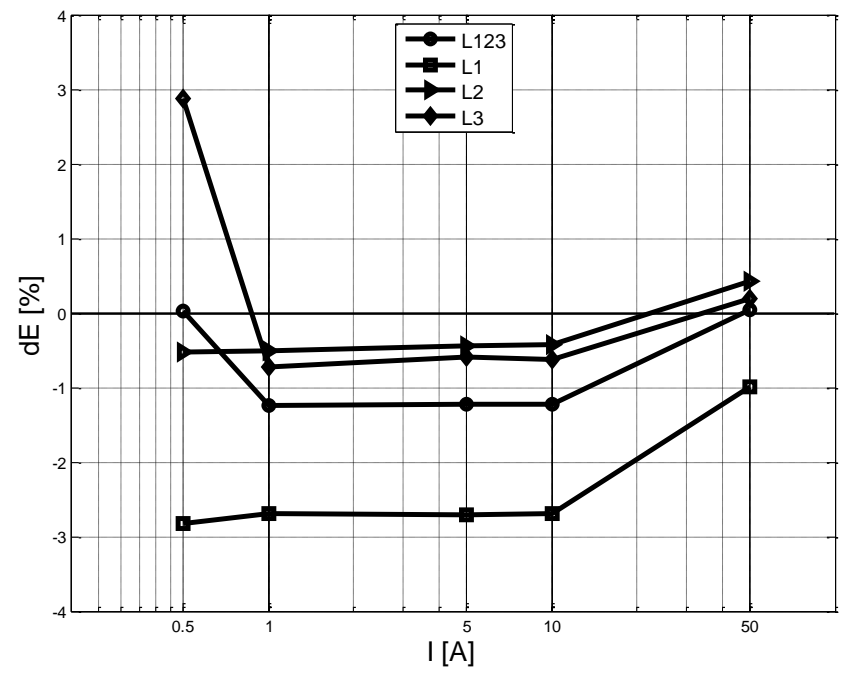

Fig. 12. The characteristics of the EEM additional error for RH7 test $(\mathrm{PF}=1 \cap$ bal. $(\mathrm{L} 123) \cap$ unbal. $(\mathrm{L1}, \mathrm{L} 2, \mathrm{~L} 3))$.

Fig. 13 shows the EEM additional error for ten random signals at unity power factor and balanced load. For some tests error values are close to zero (in RH1 test they are confined to the $0.6 \%$ limit). Some test results have positive values (in RH6 test they are confined to the $+2.8 \%$ limit) and some have negative values (in RH7 test they are confined to the $-1.0 \%$ limit). The highest DUT error values are for the $6^{\text {th }}$ random waveform in RH6 test. 


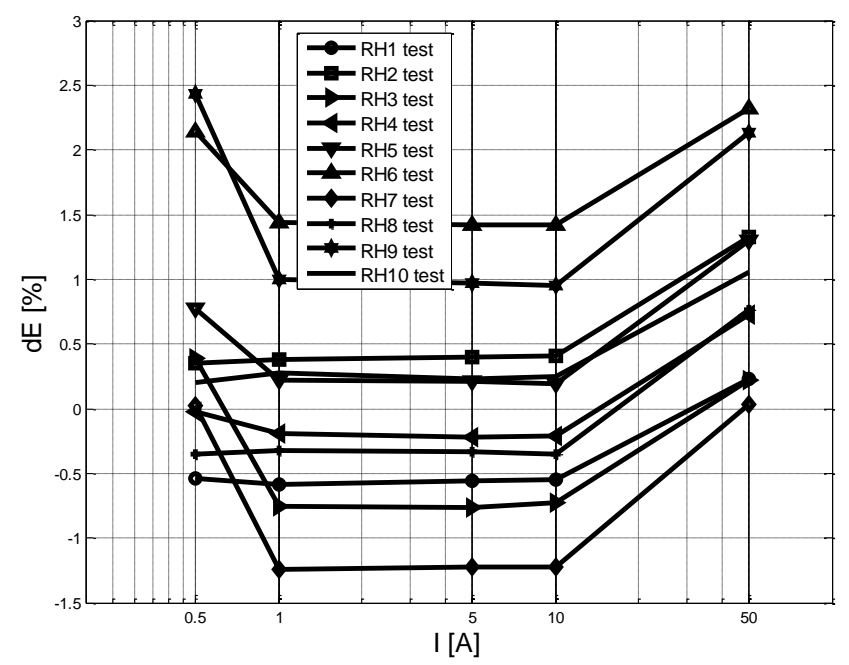

Fig. 13. The characteristics of the EEM additional error for ten standard signals ( $P F=1 \cap$ bal.).

Fig. 14 shows the EEM additional error range for ten random $\mathrm{RH}$ tests and the EEM additional error for $5 \mathrm{H}$, PFW and BFW tests with unity power factor and balanced load. Additional errors measured in $5 \mathrm{H}$ and PFW tests are within the range set by additional errors from $\mathrm{RH}$ tests, but BFW test is out of this range. However, additional error values appointed during $5 \mathrm{H}$, PFW and BFW tests are included in range designated by absolute values of additional error range appointed in $\mathrm{RH}$ tests.

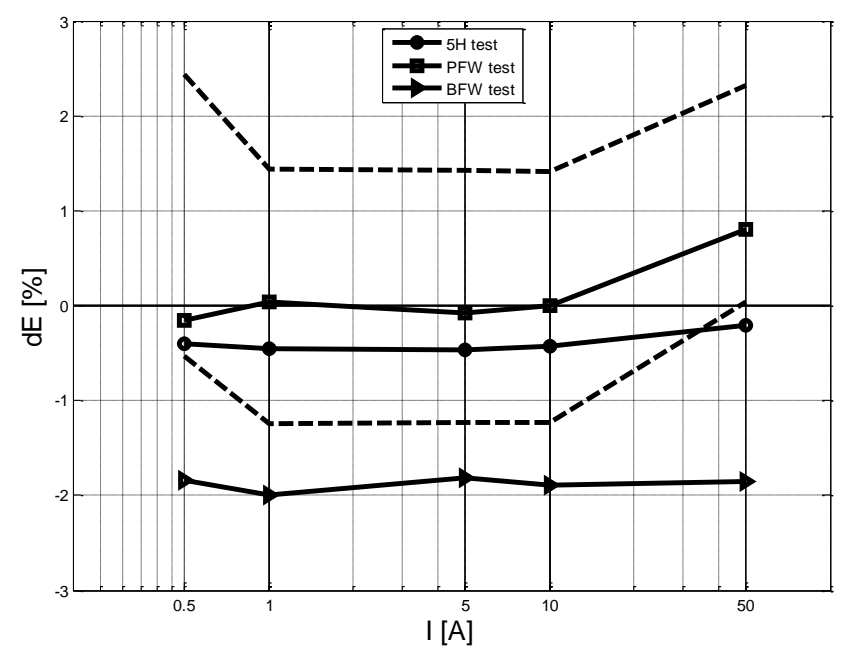

Fig. 14. The characteristics of the EEM additional error for RH tests and $5 \mathrm{H}, \mathrm{PFW}$ and $\mathrm{BFW}$ tests $(\mathrm{PF}=1 \cap \mathrm{bal}$.).

\section{SUGGESTIONS}

From the tests' results it can be seen that additional error curves are flat similarly to the current function. It means that the additional errors may be measured for one and the same current value for all tests. Therefore it is not firerequire the EEM testing at different current values (in our case 50A for $5 \mathrm{H}$ test and $5 \mathrm{~A}$ for PFW test and BFW test). Our suggestion is to test the EEM additional errors at one current value, the same for each test. The current value in such tests should be possibly high for example $0.5 \cdot I_{\max }$ (for our DUT it is $50 \mathrm{~A}$ ). If the current is higher, the EEM errors increase financial consequences in agreements between the supplier and consumer of energy. 
The EEM should be tested at balanced load. If it is necessary to get more information about the EEM additional error, the EEM should be tested for each phase at unbalanced load. Never inversely - testing the three-phase EEM only in single-phase configuration with a single-phase power source, may result in significant errors in the EEM accuracy evaluation. In order to acquire more information, the EEM can be tested for power factor equal to $0.5 \mathrm{~L}$. With this power factor value, than those ones acquired with a unity power factor.

The $N_{D}$ is a number of test points for tests performed under determined nonsinusoidal conditions (5H test, PFW test and BFW test) with balanced and unbalanced loads for two values of power factor $(\mathrm{PF}=1$ and $\mathrm{PF}=0.5 \mathrm{~L})$. In this case

$$
N_{D}=3 \text { tests } \times 1 \text { current } \times 4 \text { configurations } \times 2 P F=24 \text { points. }
$$

Because RH tests correspond with real conditions of the EEM work [9] better, it can be assumed that RH tests can replace determined tests, which means that it is necessary to carry out at least ten RH tests. In equation (8) $N_{R}$ is the number of test points performed under random nonsinusoidal conditions. If the number of RH tests will be set to 10 , then

$$
N_{R}=10 \text { tests } \times 1 \text { current } \times 4 \text { configurations } \times 2 P F=80 \text { points. }
$$

Consequently, $\mathrm{RH}$ tests application requires carrying out a test with 3 times more test points ( 80 points in $\mathrm{RH}$ tests to 24 points in determined tests) to get comparable information about the EEM additional error values. It is not known whether $10 \mathrm{RH}$ tests are enough to acquire full information about the EEM additional error values.

These studies lead us to the conclusion that testing energy meters supplying their voltage and current input channels with formerly unknown signals (RH tests), characterized by a number of randomly generated spectral components is not effective, which is in contrast to conclusions drawn by the authors in $^{13}$. Consequently, the authors of this paper suggest performing a test containing 24 points.

\section{Conclusion}

Testing electronic instruments, such as electronic energy meters (EEM), should be performed in the environment as similar to the actual conditions of their operation as possible. Three-phase nonsinusoidal conditions testing are devoted to such electronic instruments measuring. The best shapes of testing signals for the EEM calibration are sought in literature. Another proposition is to use randomly distorted waveforms for current and voltage signals.

There is a solution put forward to test the EEM accuracy error under real working conditions based on the generation of determined and randomly distorted waveforms of voltage and/or current signals according to the EN 50470. They determine the EEM testing conditions. Authors propose a test system to carry out the EEM additional error tests in different conditions - balanced and unbalanced configurations and in full current range.

It is described in this paper how to calculate the expanded uncertainty of the test system taking into account the uncertainty type $\mathrm{A}$ and $\mathrm{B}$ and energy measurement time. The best expanded uncertainty $(\mathrm{k}=2)$ of the system with reference to the power calibrator is estimated to be lower than $0.052 \%$ when measurement time is equal to $3 \mathrm{~s}$. The authors recommend taking into account the maximum value of standard deviation in the uncertainty calculation.

The experimental results of the EEM testing depending on the testing signal waveform (determined 5H, PFW and BFW tests, and randomly distorted RH test), current value, power factor value (unity and $0.5 \mathrm{~L}$ ) and the load type (balanced or unbalanced load) have been 
presented also in this paper. Additionally, it includes suggestions on how to acquire information about the EEM additional error effectively.

Therefore, Authors final conclusion is that the EEM should be tested under nonsinusoidal conditions for one current value equal to $50 \%$ of the maximum current, for balanced and unbalanced loads at unity and $0.5 \mathrm{~L}$ power factor for determined waveforms described in the EN 50470 - the use of randomly generated waveforms is not effective.

\section{References}

[1] Lin, T., Domijan, A. (October 2005). On power quality indices and real time measurement. IEEE Transactions on Power Delivery. vol. 20, No. 4.

[2] Directive 2004/22/EC of the European parliament and of the Council, Official Journal of the European Union, 2004.

[3] Salmeron, P., Herrera, R. S., Valles, A. P., Prieto, J. (April 2009). New distortion and unbalance indices based on power quality analyzer measurements. IEEE Transactions on Power Delivery. vol. 24, No. 2.

[4] Cataliotti, A., Cosentino, V., Lipari, A., Nuccio, S. (2009). Metrological characterization and operating principle identification of static meters for reactive energy: an experimental approach under nonsinusoidal test conditions. Instrumentation and Measurement. IEEE Transactions on Instrumentation and Measurement. vol. 58 , no. 5, p. 1427-1435.

[5] IEC 521: Class 0,5, 1 and 2. Alternating current watt-hour meters, 1988.

[6] Dell' Aquila, A., Marinelli M., Monopoli V. G., Zanchetta P. (July 2004) New power-quality assessment criteria for supply systems under unbalanced and nonsinusoidal conditions. IEEE Transaction on Power Delivery. vol. 19, No. 3.

[7] Caceres, R., Correa, R., Ferreyra, P., Cordero, E. (2006) Study of active electric energy meters behavior of induction and electronic types. Proc. TDC'06 IEEE PES. Caracas, Venezuela. p. 1-6.

[8] Cataliotti, A., Casentino, V., Lipari, A. Nuccio, S. (2009) On the calibration of reactive energy meters under non sinusoidal conditions. Proc. XIX IMEKO World Congress. Lisbon, Portugal. 719-723.

[9] EN 50160: Voltage characteristics of electricity supplied by public distribution networks. 1999.

[10] EN 61000-3-2: Limits for harmonic current emissions (equipment input current $\leq 16$ A per phase). 2006.

[11] EN 50470-3: Electricity metering equipment (a.c.). part 3: Particular requirements - Static meters for active energy (class indexes A, B and C). 2006.

[12] Ferrero A. Muscas C. (Apr.2000) On the selection of the best test waveform for calibrating electrical instruments under nonsinusoidal conditions. IEEE Transactions on Instrumentation and Measurement. vol. 49. no. 2, p.382-387.

[13] Ferrero, A., Faifer, M. Salicone, S. (Sep.2009) On testing the electronic revenue energy meters. IEEE Transactions on Instrumentation and Measurement. vol. 58, no. 9, p. 3042-3049.

[14] Olencki, A. (Nov. 2011) The repeatability as a quality accuracy test standard of the electrical energy meter. Electrical Review. vol. 87 no. 11, p. 154-158. http://pe.org.pl/abstract_pl.php?nid=5296.

[15] EM03 Electronic Watt-hour Meter, Lumel, http://www.lumel.com.pl/download/Z2Z4L2x1bWVsL2VuL2R1ZmF1bHRfbXVsdGlsaXN0YV9wbGlrb 3cudjAvNDQz/em_03_ang.pdf. 